\title{
1 Funktion und Geschichte des Wertfreiheitsideals
}

\subsection{Wertfreiheit und Objektivität}

Eine einfache Vorstellung von Wissenschaft lautet wie folgt: Wissenschaft liefert uns, im Gegensatz etwa zu Religion oder Politik, allgemeingültige Erkenntnisse. Ermöglicht wird dies durch ihre objektive Vorgehensweise, die darin besteht, Theorien aufzustellen und deren Gültigkeit empirisch zu überprüfen. Das Ergebnis dieser Prüfung, sprich die Akzeptanz oder Zurückweisung von Theorien, ist unabhängig von Ansichten darüber, wie die Welt beschaffen sein sollte. Ein Einfluss solcher Ansichten stellt eine mögliche Fehlerquelle dar, die methodisch kontrolliert werden kann und muss.

Eine solche Auffassung dessen, was Wissenschaft ist oder leisten kann, wird in den letzten Jahrzehnten zunehmend in Frage gestellt, sei es in der öffentlichen Wahrnehmung und Diskussion von Wissenschaft oder in der professionellen Wissenschaftstheorie. Die Entwicklung der letzteren hat gezeigt, wie schwierig eine kohärente Ausformulierung des obigen Bildes und wie fraglich einerseits seine empirische Angemessenheit, andererseits seine normative Fruchtbarkeit ist. Dennoch bestimmen Elemente dieses Bildes weiterhin unsere Vorstellung von Wissenschaft. Dies gilt insbesondere für die Annahme, dass die Wertfreiheit der Wissenschaft eine Voraussetzung für ihre Objektivität darstellt. Objektivität wiederum bildet den Kern des wissenschaftlichen Anspruchs auf epistemische Integrität und Autorität. Die Wertfreiheit der Wissenschaft lässt sich entsprechend als einer der Grundpfeiler dessen auffassen, was der Wissenschaft überhaupt erst Wert verleiht, und ist damit entscheidend für ihren gesellschaftlichen Status sowie für ihr Selbstverständnis. Auch wenn häufig zugestanden wird, dass Forschung nicht immer wertfrei ist, wird doch an der Idee, dass sie es sein sollte, oftmals festgehalten. Wertfreiheit nicht als empirische These, sondern als normatives Ideal im Sinne einer regulativen Idee, auf welche die Wissenschaft zustrebt und zuzustreben hat, wurde trotz aller Kritik keineswegs kampflos aufgegeben. 
Und obwohl es unmöglich ist, die Arbeit an der Wissenschaft von außerwissenschaftlichen Anwendungen und Wertungen frei zu halten, so ist es eine der Aufgaben der wissenschaftlichen Kritik und der wissenschaftlichen Diskussion, die Vermengung der Wertsphären zu bekämpfen, und insbesondere außerwissenschaftliche Wertungen aus den Wahrheitsfragen herauszuhalten. [...] Die Reinheit der reinen Wissenschaft ist ein Ideal, das vermutlich unerreichbar ist, für das aber die Kritik dauernd kämpft und dauernd kämpfen muss. (Popper 1962, S. 114)

[A]lthough complete freedom from value judgments cannot be achieved, it ought to be a goal or ideal of science. (Shrader-Frechette 1991, S. 44)

There should be no compromise when it comes to the proposal that ideological factors be invited into the Context of Justification [...]. We should make every attempt to keep politics and religion out of the laboratory. We may not always be successful, but that simply means that we should try harder, not that we should give up the attempt. (Koertge 2000, S. 53)

Diese Reihe exemplarischer Zitate ließe sich problemlos fortsetzen. Auch als normatives Ideal verstanden, wird die Wertfreiheit der Wissenschaft jedoch kritisiert und durch alternative Ansätze, wie etwa Philip Kitchers Well-Ordered-Science oder Helen Longinos Social Value Management, zu ersetzen versucht. Die Angemessenheit und Fruchtbarkeit dieser Alternativen sowie des traditionellen Wertfreiheitsideals zu beurteilen, setzt dabei zunächst die Klärung verschiedener Zusammenhänge voraus. Was genau beinhaltet die Forderung nach Wertfreiheit? Inwieweit wird diese Forderung von der Wissenschaft (oder einzelnen Wissenschaften) tatsächlich erfüllt? Ist es überhaupt möglich, dieses Ideal zu verwirklichen? Was sind im Einzelnen die Argumente gegen die Möglichkeit einer hinreichenden Annäherung, und wie schlagkräftig sind diese? Was bedeutet die Kritik an der Wertfreiheit für die Objektivität der Wissenschaft?

In diesem Kapitel soll zunächst ein kurzer Blick auf die Beziehung zwischen Wertfreiheit und Objektivität geworfen und erläutert werden, wie sich prinzipiell ein Objektivitätsverständnis formulieren ließe, das Wertfreiheit zumindest nicht zwingend voraussetzt. Anschließend wird die historische Formierung des Wertfreiheitsideals skizziert, um bestimmen zu können, welche Elemente des Ideals in der gegenwärtigen Diskussion um Wissenschaft und Werte verhandelt werden.

Zwischen den Vorstellungen von Wertfreiheit und Objektivität der Wissenschaft besteht, wie bereits angesprochen, ein enger Zusammenhang. 
Prima facie ist in vielen Diskussionen um Werte in der Wissenschaft die Annahme identifizierbar, dass Wertfreiheit eine notwendige, jedoch nicht hinreichende Bedingung für Objektivität darstellt. Wertbeladene Wissenschaft kann nicht objektiv sein; Wertfreiheit allein garantiert jedoch noch nicht Objektivität, da diese auch durch andere Fehlerquellen bedroht sein kann, z. B. durch ungenaues Arbeiten oder die Verwendung ungeeigneter Methoden. Diese Fehlerquellen unterscheiden sich von Werteinflüssen dadurch, dass sie zwar zu inkorrekten Ergebnissen führen, nicht aber zu systematisch verzerrten Ergebnissen, die mit bestimmten Präferenzen der Wissenschaftlerinnen übereinstimmen.

Diese Annahme von Wertfreiheit als Voraussetzung für Objektivität findet sich z. B. in den Science Wars und den Auseinandersetzungen zwischen Wissenschaftsphilosophie und der Soziologie wissenschaftlichen Wissens häufig auf beiden Seiten wieder: Objektivität und epistemische Autorität der Wissenschaft stehen und fallen mit ihrer Wertfreiheit. In der feministischen und sozialen Erkenntnistheorie hingegen wird diese Annahme im Hinblick darauf diskutiert, ob sich adäquate Konzeptionen von Objektivität entwickeln lassen, die mit (bestimmten Formen von) Werteinflüssen vereinbar sind (beispielsweise Sandra Hardings strong objectivity im Rahmen der Standpunkttheorie oder Helen Longinos social objectivity im Rahmen des kontextuellen Empirismus; vgl. z. B. Harding 1991, 1992; Longino 1990). Diese Ansätze setzen somit die Möglichkeit voraus, den Begriff der Objektivität von der Forderung nach Wertfreiheit zu entkoppeln. Das vorliegende Unterkapitel soll diese Möglichkeit der Entkoppelung plausibilisieren.

Auf einer allgemeinen Ebene drückt der Begriff der Objektivität den gängigen Intuitionen zufolge aus, dass wissenschaftliches Wissen nur das enthält, was wirklich (wahr) und unabhängig von Vorstellungen darüber ist, was wünschenswert wäre; gesichert wird dieser Status durch kontrolliertes, methodisches Vorgehen bei der Produktion wissenschaftlichen Wissens. Insofern Objektivität dabei im Gegensatz zu Subjektivität definiert wird und Werte als etwas Subjektives aufgefasst werden, liegt die Forderung nach Wertfreiheit nahe. ${ }^{1}$ Die Idee der (objektiven) Methode, die

\footnotetext{
${ }^{1}$ Hier soll nur eine verbreitete Auffassung charakterisiert, nicht aber eine bestimmte Position zur Begründung und Begründbarkeit von Werten bezogen werden. Während
} 
zu objektiven Ergebnissen führt, begründet traditionell den Anspruch der Wissenschaft auf epistemische Autorität und unterscheidet sie von anderen Modi der Wissens- oder Glaubensproduktion, wie z. B. Religion, politischen Ideologien oder auch Alltagswissen. Der Begriff der Objektivität kann dabei verschiedenen Dingen zugeschrieben werden: den Produzenten wissenschaftlichen Wissens (seien es Individuen oder Gemeinschaften), dem Prozess seiner Produktion (durch wissenschaftliche Methoden), oder dem Produkt dieses Prozesses (den unparteiischen, allgemeingültigen Resultaten). Die Verwendung des Adjektivs objektiv gibt allein noch keine Auskunft darüber, auf welche dieser drei Ebenen Bezug genommen, was genau dieser zugeschrieben und welcher Zusammenhang der Ebenen angenommen wird. Objektivität ist ein umfassender Begriff, der eine Vielzahl von Aspekten beinhaltet oder bedeuten kann, zwischen denen häufig nicht ausdrücklich unterschieden wird. Wie Heather Douglas schreibt: "The terms 'objectivity' and 'objective' are among the most used yet ill-defined terms in the philosophy of science and epistemology" (Douglas 2004, S. 453). In den letzten Jahren hat die Vielschichtigkeit dieses Begriffs jedoch verstärkt Aufmerksamkeit gefunden und wurde Gegenstand notwendiger Klärungsversuche, sowohl in Hinsicht auf seine historische Entwicklung als auch im Sinne einer systematischen Differenzierung verschiedener Verwendungsweisen.

Bezüglich der historischen Aufarbeitung des Konzepts der Objektivität ist insbesondere Lorraine Daston zu nennen, die diesen Begriff als das Ergebnis einer komplexen und kontingenten Entwicklung begreift. Das heute noch dominante Verständnis von Objektivität als Aperspektivismus ${ }^{2}$ setzte sich ihr zufolge als Ideal in den Naturwissenschaften erst im 19. Jahrhundert durch, und zwar vor allem als Reaktion auf veränderte organisatorische Bedingungen der wissenschaftlichen Arbeit. Während zuvor die individuellen Fähigkeiten eines Wissenschaftlers im Mittelpunkt gestanden

einige Autoren argumentieren, dass die Legitimität von Werteinflüssen in der Wissenschaft die Objektivität von Werten voraussetzt (siehe z. B. Rudner 1953, Smith 2004), verfährt die vorliegende Arbeit unabhängig von bestimmten Sichtweisen des ontologischen oder epistemologischen Charakters von Werten.

${ }^{2}$ Siehe dazu auch Fine (1998), der als prominente Vertreter des aperspektivistischen Objektivitätsverständnisses etwa Thomas Nagels view from nowhere (Nagel 1986) oder Bernard Williams absolute conception (Williams 1985) nennt. 
hätten und Kommunikation hochselektiv gewesen sei, d. h. vor allem in Gesprächen oder schriftlicher Korrespondenz unter persönlichen Bekannten bestanden habe, sei es etwa ab Mitte des 19. Jahrhunderts (auch aufgrund technischer Neuerungen und Verbesserungen der kommunikativen Möglichkeiten) zu einer Expansion der wissenschaftlichen Kommunikation gekommen. Die wissenschaftliche Gemeinschaft habe sich nicht mehr auf persönlich miteinander bekannte Individuen beschränkt, sondern begonnen, räumliche wie zeitliche Grenzen zu überschreiten. In diesem Prozess der Depersonalisierung der Kommunikation sei ein Verständnis von Objektivität entstanden, das sich vor allem durch die Abstraktion von individuellen Besonderheiten definiert habe. Nicht mehr der begabte, kenntnisreiche Einzelne, sondern der austauschbare Beobachter sei nun zum Idealbild der Wissenschaft geworden. Forschung habe unabhängig von bestimmten Forschenden wiederholbar und, vor allem, kommunizierbar sein müssen:

Only in the middle decades of the nineteenth century was aperspectival objectivity imported and naturalized into the ethos of natural sciences, as a result of a reorganization of scientific life that multiplied professional contacts at every level, from the international commission to the well-staffed laboratory. Aperspectival objectivity became a scientific value when science came to consist in large part of communications that crossed boundaries of nationality, training, and skill. Indeed, the essence of aperspectival objectivity is communicability, narrowing the range of genuine knowledge to coincide with that of public knowledge. (Daston 1992, S. 600) ${ }^{3}$

Diese Auffassung von Objektivität als Aperspektivismus ist wiederum selbst durch Komplexität gekennzeichnet. Elisabeth Lloyd identifiziert

\footnotetext{
${ }^{3}$ In diesem Aperspektivismus identifiziert Daston auch eine moralische Komponente in der impliziten Forderung, sich selbst und das eigene Streben nach Anerkennung hinter den Dienst an der Sache oder der Gemeinschaft zurückzustellen (vgl. ebd., S. 613 f.). Zur Geschichte des Objektivitätsbegriffs siehe beispielsweise Dear (1992); Daston/Galison (2007) in Bezug auf die Objektivität visueller Darstellungen; Porter (1992) und (1995). Anders als Daston führt Elisabeth Lloyd das aperspektivistische Verständnis von Objektivität auf die Unterscheidung zwischen primären und sekundären Qualitäten und diese letztlich auf eine religiös motivierte Ontologie zurück (vgl. Lloyd 1995). Unterschiedliche historische Herleitungen scheinen hier jedoch unproblematisch, sofern man nicht von monokausalen Prozessen ausgeht und diese sich nicht direkt widersprechen.
} 
diesbezüglich vier basale Bedeutungen. Objektiv lasse sich verstehen (1) im Sinne von unpersönlich und unparteiisch, (2) im Sinne prinzipiell öffentlicher Zugänglichkeit oder intersubjektiver Beobachtbarkeit, ${ }^{4}$ (3) im Sinne einer (von uns) unabhängigen Existenz der betreffenden Entitäten und (4) als Bezeichnung dessen, wie diese Entitäten wirklich sind. Diese Aspekte beziehen sich dabei auf verschiedene Ebenen, und zwar auf den oder die Wissenden (1); auf deren Beziehung zum Gegenstand des Wissens (2), (3); oder auf diesen Gegenstand selbst (4) (vgl. Lloyd 1995, S. 353 f.).

Arthur Fine hebt ebenfalls die zentrale Bedeutung der Unterscheidung dieser verschiedenen Aspekte hervor. Er argumentiert, dass das aperspektivistische Verständnis von Objektivität auf zwei unzulässigen Gleichsetzungen beruht oder zumindest oft mit diesen einhergeht. Erstens werde unpersönlich ("impersonal") oftmals mit unverzerrt ("unbiased") identifiziert, wofür es weder eine empirische noch eine logische Grundlage gebe, da auch eine unpersönliche Herangehensweise zu parteiischen und verzerrten Ergebnissen führen könne und oftmals führe (vgl. Fine 1998, S. 121). Die Objektivität der Produzenten, sprich der Wissenschaftler, im Sinne von Leidenschaftslosigkeit oder Neutralität, lasse noch keine Schlussfolgerungen auf die Art des produzierten Wissens zu. Zweitens werde oftmals nicht hinreichend zwischen der Objektivität des Produktionsprozesses wissenschaftlichen Wissens und der Objektivität des Produkts unterschieden, oder aber davon ausgegangen, dass erstere letztere impliziert - ein Schluss, der zumindest nicht ohne weitere Argumentation haltbar sei. Selbst methodisch angemessenes, prozedural objektives Vorgehen könne zur Produktion von Fehlern führen. Auch wenn nicht von einem objektiven Vorgehen auf objektive Geltung geschlossen werden könne, versteht Fine ersteres jedoch als die beste Möglichkeit, sich korrekten Ergebnissen anzunähern. Das bedeutet für ihn nicht, dass prozedurale Objektivität eine hohe Wahrheitswahrscheinlichkeit mit sich bringt,

\footnotetext{
${ }^{4}$ Die Idee der intersubjektiven Zugänglichkeit objektiven Wissens spielt beispielsweise bei Popper eine prominente Rolle: „Die Objektivität der wissenschaftlichen Sätze liegt darin, dass sie intersubjektiv nachprüfbar sein müssen“ (Popper 1934, S. 18). Popper versteht Objektivität dabei explizit als Merkmal des Prozesses der Produktion wissenschaftlichen Wissens, das die gegenseitige Kritik der Wissenschaftler voraussetzt - eine Auffassung, die später in der Sozialen Erkenntnistheorie insbesondere durch Longino ausgebaut wird.
} 
sondern dass dasjenige Vorgehen, welches die wissenschaftliche Gemeinschaft als bestmögliches betrachtet, die Grundlage für unser Vertrauen in die erzielten Ergebnisse bildet: "Here, objectivity is fundamentally trustmaking, not real-making" (ebd., S. 127). ${ }^{5}$

Ein Vorteil von Fines Objektivitätsauffassung als Merkmal auf der Prozessebene, das der bestmögliche Indikator für epistemische Vertrauenswürdigkeit (statt für Wahrheitswahrscheinlichkeit) auf der Produktebene ist, ist zum einen, dass diese sich nicht auf die Naturwissenschaften beschränken muss. Darüber hinaus wird der Objektivitätsbegriff hier nicht in einem absoluten Sinn durch hinreichende und notwendige Bedingungen definiert, sondern lässt Raum für eine lokale, zeitliche und disziplingebundene Variabilität normativer Standards für objektive Prozeduren. Fine selbst betrachtet, von einem pragmatistischen Standpunkt aus, diese Standards als Ergebnis eines Lernprozesses (vgl. ebd., S. 127). Damit eröffnet er argumentative Spielräume für Neukonzeptionen des Objektivitätsbegriffs in der feministischen und sozialen Erkenntnistheorie, welche Objektivität mit bestimmten Formen der Wertbeladenheit zu vereinbaren und um Aspekte der sozialen Organisation von Forschung zu erweitern versuchen.

Im Folgenden werde ich mich diesem Agnostizismus in Bezug auf die Wahrheit der Resultate objektiver Methoden anschließen und mich entsprechend vorwiegend auf die prozedurale Ebene von Objektivität konzentrieren. ${ }^{6}$ Im Sinne Fines ist dies gleichbedeutend mit der Frage danach,

\footnotetext{
${ }^{5}$ Ein Verständnis von Objektivität als allgemeingültigen, wahren Ergebnissen auf der Grundlage unparteiischer wissenschaftlicher Methoden wird dabei durchaus nicht nur in der feministischen Erkenntnistheorie kritisiert. Elisabeth Lloyd konstatiert in dieser Hinsicht jedoch einen doppelten Bewertungsmaßstab in dem Sinne, dass feministische Ansätze häufig dafür kritisiert würden, einem solchen Objektivitätsverständnis nicht zu genügen, obwohl dieses auch von nicht-feministischen Philosophen als problematisch erachtet werde (vgl. Lloyd 1995).

${ }^{6}$ Vgl. dazu auch Megill (1994), der zwischen absoluter, disziplinärer, dialektischer und prozeduraler Objektivität unterscheidet und letztere ebenfalls so versteht, dass sie von dem Ziel objektiver Wahrheiten als Ergebnis abstrahiert. Douglas (2004, 2009) differenziert wiederum zwischen acht verschiedenen Auffassungen prozeduraler Objektivität, die sie in die Kategorien Interaktionen mit der Umwelt, individuelle Gedankenprozesse und soziale Prozesse einordnet und von denen nur eine äquivalent zu der Forderung nach Wertfreiheit ist. Eine Festlegung auf eine spezifische Variante proze-
} 
welche Merkmale wissenschaftlicher Prozessen als Kriterien epistemischer Vertrauenswürdigkeit gelten können. Hierbei interessiert mich insbesondere der Zusammenhang zwischen Wertfreiheit und Objektivität, weshalb nun die relevante Idee der Wertfreiheit spezifiziert werden soll.

\subsection{Entwicklung und Elemente des Wertfreiheitsideals}

Das Ideal der Wertfreiheit hat eine lange Geschichte und umfasst verschiedene Elemente, von denen sich einige zwar in der zeitgenössischen Diskussion noch wiederfinden, andere jedoch kaum mehr explizit vertreten werden. Proctor (1999) unterscheidet in einer umfassenden historischen Untersuchung zur Formierung des Wertfreiheitsideals vier verschiedene Aspekte, welche ihm zufolge die Entwicklung des Wertfreiheitsideals entscheidend informiert haben: die Ideen der Reinheit der Wissenschaft (1), der methodischen Kontrolle (2), der Trennung von Werten und empirischen Tatsachen (3) und schließlich der Neutralität (4). Dabei betrachtet er diese Entwicklung unter Berücksichtigung einer Reihe von Auseinandersetzungen und Konflikten über das Verhältnis von Wissenschaft und Staat. In der Geschichte des Wertfreiheitsideals geht es entsprechend nicht nur um wissenschaftsinterne Debatten über geeignete normative Standards für die Akzeptanz von Theorien, sondern auch um den Status der Wissenschaft in der Gesellschaft, insbesondere immer wieder um Fragen ihrer Autonomie.

Die Idee der Reinheit der Wissenschaft (1) verfolgt Proctor zurück bis zu Platon und der antiken Bevorzugung von Theorie gegenüber Praxis, d. h. der Vorstellung, das Interesse der Wissenschaft (bzw. Philosophie) liege nicht in praktischen Belangen, sondern in dem Studium der essentiellen und ewigen Natur der Dinge (vgl. Proctor 1999, Kap. 1). Diese Vorstellung steht nach Proctor in enger Verbindung mit Fragen des sozialen Status. Reine Wissenschaft sei nur für diejenigen Personen oder Gesellschaften möglich, die nicht mehr für die Erfüllung grundlegender praktischer Bedürfnisse kämpfen müssten und sei insofern als ein Ausdruck

duraler Objektivität scheint an dieser Stelle jedoch nicht sinnvoll; wesentlich ist hier zunächst nur die Bestimmung der relevanten Ebene. 
sozialer Überlegenheit zu verstehen: "Science in the Platonic vision is the product of reflective leisure; science is the privilege of a society that has satisfied its physical needs and is free to entertain the luxury of contemplative inquiry" (ebd., S. 7). ${ }^{7}$

Ein Idealbild von Wissenschaft als reiner Kontemplation des Notwendigen, ohne jeden Bezug zur sozialen Wirklichkeit, dürfte heute kaum noch jemand vertreten. Insbesondere ist offensichtlich, dass Wissenschaft eine sehr entscheidende Rolle in der Gestaltung der gesellschaftlichen Realität einnimmt und nicht erst dann beginnt, wenn alle praktischen Probleme bereits gelöst sind. Andererseits besteht durchaus eine (weniger extreme) Idealvorstellung von Wissenschaft weiter, derzufolge diese allein oder zumindest vorrangig durch das Ziel reiner Wissensgewinnung geleitet wird. Diese Sichtweise spiegelt sich noch heute in der problematischen, jedoch verbreiteten Unterscheidung von Grundlagenforschung und angewandter Forschung (pure versus applied science) wider und geht auch hier häufig mit einer Hierarchisierung in Bezug auf die jeweilige Wertschätzung einher.

In Verbindung zur Wertfreiheit geht es bei der Idee der Reinheit darum, dass die thematische Ausrichtung der Wissenschaft sich ausschließlich an der Wissensvermehrung orientieren und von anderen Interessen, z. B. finanzieller oder politischer Natur, unabhängig sein soll. Diese Forderung ist offensichtlich überzogen und wurde schon von Weber dahingehend zurückgewiesen, dass die Selektion von Themen oder Teilaspekten

\footnotetext{
${ }^{7}$ Diese Darstellung ist allerdings etwas simplifizierend. Zunächst einmal lässt sich der antike Diskurs über Theorie und Praxis nicht eindeutig in ein heutiges Verständnis des Unterschieds zwischen grundlegender Theorie und deren praktischer Anwendung übersetzen. Bei Platon und Aristoteles ist diese Unterscheidung zuvorderst auf das Verhältnis von Philosophie und Politik ausgerichtet und steht im Zusammenhang mit Vorstellungen über unterschiedliche Formen der Lebensführung (die allerdings durchaus hierarchisierend gewertet werden) und der ethischen Frage nach dem guten Leben. Insofern als sich etwa bei Aristoteles eine Unterscheidung zwischen reiner Theorie/ Wissen und Praxis findet, bezieht sich erstere dabei auf die Erkenntnis notwendiger erster Prinzipien, aus denen weiteres Wissen deduziert werden kann. Offensichtlich entspricht dies nicht unserer heutigen Auffassung von theoretischer Wissenschaft (vgl. dazu z. B. Parry 2007). Dennoch spielt die Unterscheidung zwischen Theorie und Praxis in ihrer historisch modifizierten Form eine wichtige Rolle für die Vorstellung einer wertfreien, reinen Wissenschaft.
} 
auch von Werten der Wissenschaftler beeinflusst wird und nicht rein sein kann (siehe unten). Zudem wurde in der sozialen Erkenntnistheorie vermehrt hinterfragt, ob eine solche Reinheit überhaupt wünschenswert ist. Wissenschaft ist heute ebenso selbst eine entscheidende gesellschaftliche Ressource, wie sie auch darauf angewiesen ist, dass die Gesellschaft ihr ausreichend Ressourcen bereitstellt. Philip Kitcher etwa argumentiert nachdrücklich dafür, die Agenda-Setzung der Wissenschaft deshalb stärker an demokratischen Prinzipien (und damit gesamtgesellschaftlichen Bedürfnissen) auszurichten, wie später noch erläutert werden soll. Wie dies bereits andeutet, steht die Forderung der Reinheit der Wissenschaft häufig im Zusammenhang mit Forderungen nach ihrer politischen Autonomie und Forschungsfreiheit. So verstanden, wären nicht zwingend alle Werteinflüsse auf die Agenda-Setzung auszuschließen, sondern nur wissenschaftsexterne. ${ }^{8}$ Oftmals wird diese Autonomieforderung damit begründet, dass eine Selbstregulierung der Wissenschaft die effizienteste Organisationsform in Bezug auf die Wissensvermehrung sei. ${ }^{9}$

Forderungen nach einer Reinheit der Wissenschaft beziehen sich vorwiegend auf ihre Autonomie, nicht so sehr auf ihre Objektivität. Die Entstehung des Wertfreiheitsideals im Sinne einer notwendigen Bedingung für Objektivität wird meist auf die wissenschaftliche Revolution im 17. Jahrhundert datiert (vgl. z. B. Lacey 1999, Kap. 1). Insbesondere durch das Werk Francis Bacons ist die Idee der Reinheit durch die der Methode (2) ergänzt worden, welche zum entscheidenden Kriterium für die epistemische Autorität der Wissenschaft wurde: Objektivität sei nur auf der Basis einer kontrollierten Herangehensweise möglich. Werteinflüsse stellen dabei eine von verschiedenen Fehlerquellen dar, die es zu eliminieren gelte und die Bacon unter den Begriff der Idole subsumiert. Bacon beschreibt den menschlichen Geist als einen Spiegel, der die empirische Wirklichkeit

\footnotetext{
${ }^{8}$ Die Forderung nach politischer Unabhängigkeit beschränkt sich dabei nicht auf die Agenda-Setzung, sondern kann auch beispielsweise die Verwendung bestimmter Methoden oder die Publikation der Resultate betreffen.

${ }^{9}$ Zur Thematik der Forschungsfreiheit vgl. Wilholt (2010, 2012), der zwischen einer epistemologischen Begründung der Forschungsfreiheit (durch das Argument der Effizienz von Selbstregulierung) und einer politischen Begründung (über die Bedeutung wissenschaftlichen Wissens für die Bildung informierter Positionen der Bürger in einer Demokratie) unterscheidet und die Tragweite dieser Begründungen untersucht.
} 
nur verzerrt abbilde, und zwar aufgrund von Ansichten, Wahrnehmungen und Vorurteilen, für die es zwar keine gesicherte Grundlage gebe, die aber dennoch auf die Wirklichkeit projiziert würden. $\mathrm{Zu}$ diesen verzerrenden Faktoren gehören die Idola Tribus (Fehler, die in der Natur und dem Wahrnehmungsapparat des Menschen begründet liegen), Idola Specus (idiosynkratische Verzerrungen), Idola Fori (geteilte Verzerrungen) und Idola Theatri (unhinterfragte philosophische Dogmen) (vgl. Bacon 1620, 39 ff.). Da diese Idole zu Fehlern in der Wahrnehmung und Interpretation der empirischen Wirklichkeit führen könnten, müsse diese Wahrnehmung mittels Experimenten kontrolliert werden, um ihren Einfluss weitgehend auszuschalten. $^{10}$

Darüber hinaus hat Bacon die antike Trennung von Theorie und Praxis als unfruchtbar und hinderlich für den wissenschaftlichen Fortschritt kritisiert. Diese Kritik steht in engem Zusammenhang mit Bacons Empirismus und der Betonung der Rolle des Experiments in der Wissenschaft. Das Ziel liegt nun nicht mehr in der reinen Kontemplation der ewigen Aspekte der Natur, sondern in deren Beherrschung. Allerdings lebt die Idee einer Reinheit der Wissenschaft Proctor zufolge noch weiter in Bacons Warnung davor, Forschung zu sehr auf kurzfristigen Nutzen auszurichten, statt nach Wissen um seiner selbst willen zu suchen. Nur letzteres führe im Endeffekt zu langfristigen epistemischen und praktischen Gewinnen: "Like happiness, utility is something found only when not sought" (Proctor 1999, S. 31). Hier findet sich eine Grundlage des epistemologischen Arguments für die Forschungsfreiheit und Autonomie der Wissenschaft wieder, dass nur die größtmögliche Freiheit es der Wissenschaft erlaube, genuine und damit unvorhersehbare Fortschritte in der Wissensgewinnung zu machen (vgl. z. B. Polanyi 1962).

Diese Verwehrung gegen externe Direktion bilde dabei die eine Seite des Royalist compromise, als welchen Proctor die Selbstbeschränkung der

\footnotetext{
${ }^{10}$ Proctor versteht die Baconschen Idole als Hindernisse für den wissenschaftlichen Fortschritt, die durch moralische Werte und Ansichten entstehen ("the progress of science being hampered by the taint of moral knowledge"; Proctor 1999, S. 31). Diese Interpretation ist jedoch $\mathrm{zu}$ eng, da es sich bei dem schädlichen Einfluss von Idolen auch um philosophische Lehren oder empirische Annahmen handeln kann, die noch nicht wissenschaftlich bestätigt wurden und die doch unseren Blick auf die Welt formen.
} 
Royal Society auf wissenschaftliche Fragen beschreibt, die den Ausschluss von Fragen der Moral, Religion oder Politik aus dem Zuständigkeitsbereich der Wissenschaft bedeutet habe. Die Royal Society habe gewisse Grenzen für die wissenschaftliche Tätigkeit akzeptiert, um sich Freiheit innerhalb dieser Grenzen zu sichern (vgl. Proctor 1999, S. 33 ff.). Wertfreiheit wird hier zu einer Bedingung für die Institutionalisierung und Professionalisierung der Wissenschaft (ein Prozess, der sich bei der Etablierung der Sozialwissenschaften wiederholt, siehe unten).

Ein weiteres fundamentales Element des Wertfreiheitsideals (und gleichzeitig die logische Grundlage obigen Kompromisses) liegt in der Trennung des Seins vom Sollen (3) und dem Ausschluss des Sollens aus dem Bereich der Wissenschaft. Während in der Antike die natürliche Ordnung gleichzeitig noch eine ethische Ordnung darstellte und Werte ein integraler Bestandteil des teleologischen Erklärungsmodells waren, war die wissenschaftliche Revolution auch eine Revolution in Bezug auf das Verständnis von Werten (vgl. Proctor 1999, Kap. 3 und 4). Der Wert von etwas liegt nun nicht mehr in der Natur der Dinge; vielmehr wird die Frage, ob und wie sich Werte begründen lassen, zu einem kontrovers diskutierten Problem. Die Einheit von Sein und Sollen weicht der modernen Unterscheidung der Wertsphäre von der empirischen Sphäre im Sinne einer gegenseitigen kausalen und logischen Unabhängigkeit. Gegenstand der Wissenschaft ist das empirisch Gegebene, welches sich weder aus Wertvorstellungen heraus erklären lässt, noch zu bestimmen vermag, wie die Welt sein sollte. Die Separierung des Deskriptiven vom Normativen drückt damit zunächst eine ontologische These über die fundamentale Unterschiedlichkeit der betreffenden Gegenstandsbereiche und ihre jeweilige Eigenständigkeit aus. Dieser Position entspricht die logische These, die sich in David Humes berühmter erster Formulierung des naturalistischen Fehlschlusses ausdrückt:

In every system of morality, which I have hitherto met with, I have always remark'd, that the author proceeds for some time in the ordinary way of reasoning [...] when of a sudden I am surpriz'd to find, that instead of the usual copulations of propositions, is, and is not, I meet with no proposition that is not connected with an ought, or an ought not. This change is imperceptible; but is, however, of the last consequence. For as this ought, or ought not, expresses some new relation or affirmation, 'tis necessary [...] that a reason should be 
given, for what seems altogether inconceivable, how this new relation can be a deduction from others, which are entirely different from it. (Hume 1739, S. 469).

Normative Aussagen unterscheiden sich in logischer Hinsicht von deskriptiven, weshalb keine Folgerungsbeziehungen zwischen ihnen möglich sind. Weder folgen empirische Aussagen aus Vorstellungen darüber, was wünschenswert wäre, noch vermögen empirische Erkenntnisse normative Positionen zu begründen. Damit hängt auch die Vorstellung von Neutralität (4) als Teil des Wertfreiheitsideals zusammen: das Sein begründet kein Sollen. Deshalb sollten Wissenschaftler Abstand davon nehmen, normative Positionen als wissenschaftlich gesichert zu proklamieren - hierbei kann es sich nur um einen logischen Fehlschluss handeln. ${ }^{11}$ Darüber hinaus ist der Aspekt der Neutralität jedoch mit dem Problem der Verantwortung für die gesellschaftlichen Konsequenzen von Wissenschaft verbunden und der Frage, ob sich diese durch den Hinweis auf die Wertfreiheit der Forschung zurückweisen und dem Bereich der Politik zuschreiben lässt - in dem Sinne, dass Wissenschaft Wissen generiert, über dessen Verwendung andere entscheiden und gegebenenfalls auch zur Rechenschaft zu ziehen sind. Diese stärkere Neutralitätsforderung zielt also nicht auf die Vermeidung von Fehlschlüssen, sondern auf eine vollständige (moralische) Autonomie der Wissenschaftler in Bezug auf etwaige Folgen ihrer Forschung.

Dieser Aspekt des Wertfreiheitsideals ist zumindest fragwürdig und wird oft vehement kritisiert. Auch wenn sich normative Urteile nicht logisch aus empirischen ableiten lassen, sind doch häufig Debatten, die Wertfragen berühren, mit deskriptiven Prämissen verknüpft; man denke etwa an die Kontroversen um den Kreationismus oder an Studien zu Intelligenzunterschieden zwischen den Geschlechtern. Empirische Untersuchungen, die beispielsweise Frauen geringere mathematische Fähigkeiten zuschreiben, haben in unserem gegebenen gesellschaftlichen Kontext sehr wohl Auswirkungen auf Wertkonflikte, etwa um Fragen der Gleichberechtigung, und damit unter Umständen erhebliche soziale Konsequenzen. Wie gesagt, handelt es sich dabei nicht um logische Folgerungsbeziehungen.

${ }^{11}$ Diese schwache Form von Neutralität lässt sich im Grunde auf die logische Trennung von Sein und Sollen (3) reduzieren, nur ist die Beziehung hier umgekehrt: während Objektivität davon abhängt, dass nicht vom Sollen auf das Sein geschlossen wird, ist Neutralität dadurch bedingt, dass nicht vom Sein auf das Sollen geschlossen wird. 
Selbst wenn sich Frauen eine geringere mathematische Intelligenz zweifelsfrei nachweisen ließe, folgt daraus nicht, dass ihnen deshalb eine geringere Wertschätzung zukommen und diese sich auch in ihrem sozialen Status niederschlagen sollte. Welche gesellschaftlichen Konsequenzen diese empirischen Ergebnisse haben, ist abhängig davon, welche Strukturen und Werthaltungen in einem sozialen Kontext bereits gegeben sind, und wäre anders in einer Gesellschaft, die mathematischen Fähigkeiten keinen oder nur geringen Wert beimisst. Da Wissenschaft jedoch nicht in einem rein logischen Raum, sondern immer in einem spezifischen gesellschaftlichen Kontext operiert, sind ihre Ergebnisse (oder auch bereits die Themenwahl) praktisch keineswegs neutral in Hinsicht auf normative Fragen. ${ }^{12}$

Neben den Auswirkungen auf Werthaltungen und entsprechende Konflikte ist zudem die entscheidende Rolle zu betonen, welche die Wissenschaft für die Gestaltung der sozialen Wirklichkeit durch ihre praktische Anwendung und technologische Umsetzung hat. Insbesondere seit dem 20. Jahrhundert wird Wissenschaft nicht mehr nur als glorreiche Errungenschaft der Menschheit gesehen, sondern sind verstärkt auch die mit ihr verbundenen Gefahren in den Blick geraten. Die Rolle der Chemie im Ersten Weltkrieg, die Entwicklung der Atombombe im zweiten Weltkrieg, die möglichen Implikationen genetischer Manipulation oder auch die problematische Distribution medizinischer Forschung haben Anlass zu einer Reihe von Kritiken an der Idee einer autonomen und neutralen Wissenschaft als sozial unverantwortlich gegeben (vgl. z. B. Proctor 1999, Kap. 17; Kitcher 2001, 2004; Longino 1990, 1996; Forge 2008; Douglas 2000, 2009).

Im Folgenden soll in Bezug auf die dargestellten vier Aspekte des Wertfreiheitsideals geklärt werden, inwieweit und in welcher Ausformulierung diese in der heutigen Debatte verhandelt werden und für die vorliegende Arbeit relevant sind. Um die gegenwärtige Form des Wertfreiheits-

\footnotetext{
${ }^{12}$ Noretta Koertge weist darauf hin, dass die normativen Konsequenzen wissenschaftlicher Forschung oftmals aufgrund der Komplexität relevanter politischer Kontexte kaum vorhersagbar sind (vgl. Koertge 2000, S. 54). Dieses Problem soll hier nicht bestritten werden. Die Frage ist jedoch, zu welcher Reaktion dies führen sollte $-\mathrm{zu}$ einer größeren Anstrengung seitens der Wissenschaft, diese Kontexte zu erfassen, oder zu einem Rückzug auf wissenschaftliche Neutralität und einer damit einhergehenden Übergabe der Verantwortung an die Politik.
} 
ideals zu bestimmen, werde ich zunächst die Entwicklung dieser Aspekte an zwei diesbezüglichen Meilensteinen beleuchten: die Verteidigung der Wertfreiheit durch Max Weber und die Beschränkung von Wissenschaft auf Logik und Erfahrung im Neopositivismus.

\subsection{Max Weber und der deutsche Werturteilsstreit}

Der Werturteilsstreit unter den Mitgliedern des Deutschen Vereins für Socialpolitik, in dessen Kontext Max Weber die Wertfreiheit verteidigte, stellt die früheste explizite Kontroverse über dieses Ideal dar. Webers Überlegungen sind dabei im Lichte der im 19. Jahrhundert entstehenden Sozialwissenschaften zu sehen und stehen in Relation zu der Frage, wie sich die Sozialwissenschaften zu den Naturwissenschaften einerseits und zur Politik andererseits verhalten. Angesichts des immensen Erfolgs der Naturwissenschaften seit der wissenschaftlichen Revolution im 17. Jahrhundert - sowohl was die Lösung theoretischer Probleme als auch die technologische Umsetzung von Erkenntnissen betrifft - standen die Sozialwissenschaften unter einem gewissen Legitimierungsdruck: Konnte ihnen der gleiche Status von Wissenschaft zugesprochen werden? Waren ähnliche Erfolge wie in den Naturwissenschaften zu erwarten, und wie würde man diese erreichen? War Soziologie als soziale Physik zu verstehen, wie Auguste Comte es tat, oder gab es einen grundsätzlichen Unterschied zwischen Sozial- und Naturwissenschaften?

Einerseits wird in diesem Kontext der Etablierung der Sozialwissenschaften die Wertfreiheit zum Maßstab ihrer Wissenschaftlichkeit: "Social Scientists proclaimed their work neutral as part of an attempt to make that work scientific" (Proctor 1999, S. 66). Gleichzeitig erfüllt die Berufung auf Wertfreiheit die Funktion, die Autonomie der Sozialwissenschaften - trotz ihres thematischen Bezugs zur sozialen Wirklichkeit - vor politischer Einflussnahme zu sichern und sich von Forderungen des Staates oder dominanter gesellschaftlicher Strömungen wie dem Sozialismus, dem Nationalismus oder dem Feminismus unabhängig zu machen. (vgl. dazu ebd., S. 70 sowie S. 99-120). 
Den Hintergrund des Werturteilsstreits bildet der Methodenstreit in der Nationalökonomie, der sich zwischen der abstrakt-theoretischen Schule (prominent vertreten von Carl Menger) und der historischen Schule (prominent vertreten von Gustav Schmoller) zutrug. Die theoretische Schule plädierte für einen methodischen Monismus der Sozial- und Naturwissenschaften. Angesichts des Erfolgs der letzteren solle die Nationalökonomie deren Verfahrensweisen übernehmen und nach allgemeinen Gesetzen suchen, aus denen sich die sozialen Phänomene deduzieren und so erklären ließen. Die historische Schule hingegen vertrat den Standpunkt, dass die Sozialwissenschaften einer gesonderten Methodologie bedürfen. Ihr Interesse liege weniger darin, allgemeine Gesetze zu erkennen, als vielmehr konkrete historische Ereignisse $\mathrm{zu}$ verstehen und $\mathrm{zu}$ beschreiben. ${ }^{13}$ Max Weber war ein Gegner des Methodenmonismus der abstrakt-theoretischen Schule und hatte seine Ursprünge in der historischen Schule, an der er jedoch die fehlende Trennung empirischer und wertender Urteile bemängelte (vgl. Drysdale 2007, S. 33). Die ältere Generation der historischen Schule (insbesondere Schmoller) vertrat die Auffassung, dass ökonomische Begriffe wie etwa Produktivität essentiell wertende Begriffe seien und sein müssten. ${ }^{14}$ Dies sei jedoch nicht problematisch aufgrund der objektiv erfassbaren Natur von Werten. Im Gegenteil sei es die Aufgabe der Sozialwissenschaftler, in politischen Fragen eine führende Rolle einzunehmen - eine Auffassung, die schon mit der schwachen Neutralitätsforderung

${ }^{13}$ Als eine wichtige Quelle ist hier der Heidelberger Neo-Kantianismus zu erwähnen und Wilhem Windelbands Differenzierung von Kultur- und Naturwissenschaften aufgrund ihres abweichenden Erkenntnisinteresses und eines entsprechenden Unterschiedes in ihrer Methodologie. Während die Naturwissenschaften ihm zufolge nomothetisch verfahren und ihren Gegenstand mittels allgemeiner Gesetze erklären, seien die Kulturwissenschaften idiographisch ausgerichtet und versuchten, die betreffenden Phänomene in ihrer individuellen Besonderheit zu erfassen.

${ }^{14}$ Ein Argument, dass auch in der heutigen Philosophie noch diskutiert und vertreten wird; vgl. etwa Dupré (2007), der argumentiert, dass es unmöglich sei, nicht-wertbeladene Begriffe z. B. von ökonomischer Produktivität oder Vergewaltigung zu bilden. Ein weiteres Beispiel für die Wertbeladenheit von Begriffen sind Vorstellungen von Gesundheit und Krankheit (siehe dazu Kap. 6). Diesbezüglich wird noch zu diskutieren sein, was eine derartige Wertbeladenheit von Begriffen für die Möglichkeit der Wertfreiheit betreffender Theorien bedeutet. 
kollidiert und ihren Vertretern die Bezeichnung als Kathedersozialisten eingebrachte (vgl. Proctor 1999, S. 87 ff.).

Weber wendet sich entschieden gegen diese Position und kritisiert die politische Stellungnahme im Namen der Wissenschaft als intellektuelle und moralische Unredlichkeit, da er davon ausgeht, dass Wertauffassungen letztlich etwas rein Subjektives seien und die Wissenschaft entsprechend keine besondere Autorität habe, um über politische Fragen Auskunft zu geben. Zwar spreche nichts gegen ein gesellschaftliches Engagement von Wissenschaftlern in ihrer Rolle als Bürgern, diese sei dann jedoch von der Rolle des Wissenschaftlers strikt zu trennen. Weber richtet sich vehement gegen politische Stellungnahmen, die durch den Deckmantel der Wissenschaftlichkeit gegen Kritik immunisiert werden (vgl. Weber 1917, 1919).

Webers Entwurf einer verstehenden, jedoch wertfreien und objektiven Soziologie ist als ein Lösungsversuch des Methodenstreits in der Nationalökonomie zu sehen, welcher der Soziologie den Status einer Wissenschaft zusichert, ohne einen Methodenmonismus anzunehmen. Seine Auffassung der angemessen Methode in der Soziologie lässt sich dabei weder als rein nomologisch noch als rein idiographisch bezeichnen. Einerseits reiche die Reduktion der sozialen Wirklichkeit auf allgemeine Gesetze nicht aus, um diese in ihrer individuellen Besonderheit und Bedeutung hinreichend zu verstehen. Andererseits lasse sich ein solches Verständnis auch nicht durch eine bloß deskriptive Reproduktion der einzelnen Begebenheiten erzielen, da unsere Auffassung der sozialen Wirklichkeit immer eine theoretisch vermittelte sei. In Bezug auf die Eigentümlichkeit der Begriffsbildung in der Soziologie entwickelt Weber das Konzept des Idealtypus (vgl. dazu Weber 1904). Diese Typisierung sozialer Phänomene gehe dabei von der gedanklichen Steigerung bestimmter, relevant erscheinender Aspekte aus. Deren Relevanz erkläre sich aber nicht daraus, dass diese Aspekte essentielle Merkmale repräsentierten, sondern aus der Bedeutung, die wir ihnen aus einer bestimmten Perspektive heraus verliehen.

Es gibt keine schlechthin ,objektive' wissenschaftliche Analyse des Kulturlebens oder [...] der ,sozialen Erscheinungen' unabhängig von speziellen oder ,einseitigen' Gesichtspunkten, nach denen sie [...] als Forschungsprojekt ausgewählt, analysiert und darstellend gegliedert werden. (Ebd., S. 170)

Um den spezifischen Charakter der Objektivität in den Sozialwissenschaften zu verstehen, ist es nach Weber wichtig, ihren Bezug zu Wertideen als 
Voraussetzung für die Konstitution und Strukturierung der sozialen Wirklichkeit zu erkennen. Darüber hinaus sei die Rolle dieser Wertideen bei der Bildung von idealtypischen Begriffen $\mathrm{zu}$ beachten, mittels derer diese Wirklichkeit über ihre Abweichung von diesen Idealtypen empirisch erfassbar werde. Die Objektivität der Sozialwissenschaft werde nicht durch eine Imitation der Naturwissenschaften oder durch theoriefreie Deskription ermöglicht, sondern durch die Verwendung dieser spezifisch sozialwissenschaftlichen Form der Begriffsbildung und darauf aufbauenden Hypothesenbildung. Andererseits sei eine notwendige Bedingung für Objektivität, dass der Einfluss der Wertideen nicht dahingehend missverstanden werde, dass ihre Richtigkeit vorausgesetzt oder als Ergebnis der empirischen Forschung ausgegeben werde.

Die ,Objektivität' sozialwissenschaftlicher Erkenntnis hängt vielmehr davon ab, dass das empirisch Gegebene zwar stets auf jene Wertideen, die ihr allein Erkenntniswert verleihen, ausgerichtet, in ihrer Bedeutung aus ihnen verstanden, dennoch aber niemals zum Piedestal für den empirisch unmöglichen Nachweis ihrer Geltung gemacht wird. (Ebd., S. 214)

Festzuhalten ist, dass auch bei Weber Objektivität den Anspruch auf Wissenschaftlichkeit im Sinne epistemischer Autorität begründet. Diese Objektivität identifiziert er mit der Allgemeingültigkeit der produzierten Resultate: „das Merkmal wissenschaftlicher Erkenntnis (muss) in der ,objektiven" Geltung ihrer Ergebnisse als Wahrheit gefunden werden" (ebd., S. 147). Objektive Gültigkeit ist für ihn aperspektivistische Gültigkeit und unabhängig von sozialen Kontexten und Weltanschauungen: zwar ist die Perspektive auf soziale Phänomene eine wertgebundene, die darauf aufbauenden wissenschaftlichen Ergebnisse seien jedoch gültig in einer Weise, die auch für jemanden mit einer gänzlich anderen Perspektive hielte („einem Chinesen“ etwa; vgl. ebd., S. 155). Diese Objektivität ist einerseits abhängig von der Art und Weise der Produktion dieser Resultate, d. h. von der Verwendung einer dem sozialwissenschaftlichen Gegenstandsbereich angemessenen Methode (2). Andererseits ist eine notwendige Bedingung der Allgemeingültigkeit (und damit Objektivität) in der Trennung des Seins 
vom Sollen (3) zu sehen. Werte unterscheiden sich für Weber von Tatsachen in ontologischer Hinsicht durch ihre Subjektivität, die ihre epistemologische Irrelevanz impliziert. Webers Argumentation richtet sich hier vorwiegend gegen einen Schluss vom Sein auf das Sollen: wissenschaftliche Ergebnisse können keine politischen Positionen begründen. Da der Grund hierfür in den fehlenden Folgerungsbeziehungen zwischen Werten und Tatsachen liegt, gilt jedoch auch die umgekehrte Beziehung: die Gültigkeit wissenschaftlicher Ergebnisse kann nicht auf wertenden Vorstellungen davon beruhen, wie jemand die Wirklichkeit gerne hätte. Aufgrund der Subjektivität von Werten würde dies die Allgemeingültigkeit und damit Objektivität der Wissenschaft bedrohen.

Wissenschaft kann nach Weber allerdings Werte zu einem Gegenstand ihrer Forschung machen. Einerseits könne eine logische Analyse Werthaltungen auf grundlegende Wertaxiome zurückführen sowie die Konsistenz von verschiedenen Werten prüfen, und damit eine Grundlage für die Entscheidung des Individuums in Bezug auf die Wahl letzter Zwecke bieten. Andererseits könne sie in Bezug auf diese Zwecke empirische Antworten darauf liefern, ob sie praktisch sinnvoll sind, d. h. überhaupt erreichbar. Zudem vermöge sie bei vorgegeben Zwecken zu untersuchen, welches die geeigneten Mittel zu ihrer Erreichung sind sowie Auskunft darüber geben, welche unerwünschten Konsequenzen diese Mittel möglicherweise haben. Zum Beispiel kann die Nationalökonomie demnach (Wenn-Dann-)Aussagen darüber machen, wie eine Steigerung der wirtschaftlichen Produktivität zu erreichen ist, wenn diese als Ziel gewählt wurde, und welche Nebenwirkungen die betreffenden Maßnahmen haben könnten, etwa mögliche Unruhen in der Arbeiterschaft. Als Wissenschaft kann sie aber über das Ziel der Produktionssteigerung nicht entscheiden (vgl. ebd., S. 149 ff.). Webers Kritik an den Kathedersozialisten richtet sich hier gegen die Vermischung des Seins mit dem Sollen (bzw. die vorgebliche Folgerung des Sollens aus dem Sein) und die Aufgabe der wissenschaftlichen Neutralität (4) durch eine vorgebliche Verwissenschaftlichung der Politik. Die Sozialwissenschaften produzieren Erkenntnisse, die für politische Maßnahmen relevant sind; es sei aber nicht die Aufgabe der 
Wissenschaftler qua Wissenschaftler, über diese Maßnahmen, sprich die Anwendung ihrer Erkenntnisse zu entscheiden. ${ }^{15}$

${ }^{15}$ Die Frage nach der Möglichkeit, politische Positionen sozialwissenschaftlich zu legitimieren, steht auch im Mittelpunkt der zweiten großen Debatte um Werte in der deutschen Soziologie: dem Positivismusstreit zwischen Vertretern des Kritischen Rationalismus (Karl Popper, Hans Albert) und Vertretern der kritischen Theorie der Frankfurter Schule (Theodor Adorno, Jürgen Habermas). Der Kritische Rationalismus versteht Soziologie - in der Tradition Max Webers - als objektiv-verstehende Sozialwissenschaft mit dem Ziel, soziales Handeln zu verstehen und zu erklären. Die Objektivität einer Wissenschaft ist für Popper eine Frage der Methode, also ein Merkmal des Prozesses der Produktion wissenschaftlichen Wissens. Dieses Merkmal liegt in der gegenseitigen Kritik der Wissenschaftler, die wiederum dadurch ermöglicht wird, dass die verhandelten Aussagen empirisch falsifizierbar sind, was gleichzeitig das Poppersche Kriterium für Wissenschaftlichkeit ist. Der Prozess der kritischen Prüfung ist dabei auch als sozialer Prozess zu sehen und von sozialen Bedingungen abhängig, was jedoch für Popper nicht heißt, dass soziale Faktoren oder Werte auf das Ergebnis dieser Prüfung einwirken dürften. Wertfreiheit ist dabei als regulatives Ideal zu verstehen, dem sich Wissenschaft durch den fortwährenden Prozess der Kritik annähert.

Die Kritische Theorie hingegen versteht Soziologie als dialektische Wissenschaft (in der Tradition Hegels und Marx) mit dem expliziten Ziel der Gesellschaftskritik. Der Punkt, an dem sich die Geister scheiden, ist der Anspruch der Kritischen Theorie, einen normativen Standpunkt wissenschaftlich begründen zu können. Die dialektische Methode führe, so Adorno, zu einem Begriff der gesellschaftlichen Totalität, der dem objektiven Wesen der Gesellschaft - dem, wie sie sein soll - entspreche, und der es erst ermögliche, die seiende Gesellschaft (immer im Hinblick auf ihren Zielzustand) zu untersuchen und zu kritisieren. In diesem dialektisch erfassten Wesen der Gesellschaft fielen Wahrheit und moralische Richtigkeit zusammen: Die normative Ebene sei von der deskriptiven letztlich nicht zu trennen und die Dichotomie von Sein/Sollen eine kontingente historische Erscheinung, die es dialektisch zu überwinden gelte.

Der Kritische Rationalismus kritisiert (ähnlich wie Weber in Bezug auf die Kathedersozialisten und meines Erachtens zu recht) diese Postulierung normativer Ziele als zwar wissenschaftlich begründet, jedoch jeder wissenschaftlichen Prüfung unzugänglich. Die Frankfurter Schule immunisiere sich gegen jegliche Kritik, die nicht mit ihrer Auffassung der dialektischen Methode des historischen Materialismus - die eng verknüpft ist mit einer bestimmten politischen Position - übereinstimme. Die Kritischen Theoretiker wiederum bemängeln, dass das Ideal der wertfreien Wissenschaft zu einer Instrumentalisierung derselben und damit zu einer Festigung der jeweils bestehenden Herrschaftsverhältnisse führe (vgl. Adorno et al. 1969).

Dazu lässt sich sagen, dass das Wertfreiheitsideal, wie dargestellt, verschiedene Elemente enthält und die Annahme der Humeschen Trennung sowie die Idee der metho- 
Die erforderliche Trennung von Wissenschaft und Werten macht Weber dabei zu einer Aufgabe der Selbstbeschränkung und Gewissensforschung der einzelnen Wissenschaftlerinnen. Er räumt zwar ein, dass die Trennung von Empirie und Werten mitunter schwierig sei und dass Werthaltungen auch unbewusst etwa die Gewichtung von Argumenten beeinflussen könnten. Dennoch gelte es, sich dem Ideal der Wertfreiheit so weit wie möglich anzunähern (vgl. ders. 1917, S. 497 f.). Die Verantwortung für diese Annäherung überlässt er dabei dem Individuum im Sinne einer moralischen Pflicht: „Es ist gewiss möglich, daß es dem Einzelnen nur ungenügend gelingt, seine subjektive Sympathie auszuschalten. Dann setzt er sich der schärfsten Kritik vor dem Forum des eigenen Gewissens aus“" (ders. 1919, S. 602).

Weber nimmt hier die von Fine kritisierte Gleichsetzung verschiedener Ebenen des Objektivitätsbegriffs vor, indem er Objektivität erstens in der Gültigkeit der Produkte wissenschaftlicher Forschung lokalisiert, deren Erreichung zweitens durch die Verwendung einer angemessenen Methode sichergestellt sieht und deren korrekte Verwendung drittens zwar nicht von einer Unparteilichkeit, wohl aber der moralischen Integrität einzelner Wissenschaftler abhängig macht. Dabei führt er jedoch nicht aus, wieso davon ausgegangen werden kann, dass das methodische Vorgehen zu allgemeingültigen Wahrheiten führe oder wie die Trennung des Seins vom Sollen (bzw. die individuelle Gewissensforschung) durch wissenschaftliche Methoden (oder soziale Prozesse der Wissenschaftsgemeinschaft) gesichert oder zumindest unterstützt werden könnte. Letztlich liegt damit die Bewertung epistemischer Vertrauenswürdigkeit in einer Beurteilung der Integrität individueller Wissenschaftlerinnen, deren Erkenntnisinteressen zwar wertgeleitet sind, wodurch diese Erkenntnisse selbst aber nicht beeinflusst sein dürfen. Auf der anderen Seite erkennt Weber an, dass Werte in den Sozialwissenschaften eine entscheidende Rolle spielen, indem sie Selektions- und Strukturierungsprozesse leiten, und wendet sich damit gegen die Idee der Reinheit (1) als Element des Wertfreiheitsideals. Diese Wert-

dischen Kontrolle nicht implizieren, dass jegliche soziale Verantwortung zurückzuweisen ist; es müssen nicht gleichzeitig auch die Ideen der Reinheit und der (starken) Neutralität vertreten werden. In jedem Fall begründet aber die Kritik an der Neutralität noch nicht die Möglichkeit einer dialektischen Entdeckung des Richtigen. 
einflüsse auf die Ausrichtung von Forschung sind für Weber unproblematisch, da er annimmt, dass die Gültigkeit der Resultate von diesen anfänglichen Einflüssen unabhängig ist - ein Gedanke, der seine Ausbuchstabierung in der neopositivistischen Unterscheidung von Entdeckungs- und Rechtfertigungskontext findet.

\subsection{Wertfreiheit im Neopositivismus}

Das klassische Bild des Neopositivismus ist eines, in welchem dieser - in seiner Konzentration auf Logik und Erfahrung als einzig legitimen Einflüssen auf die Bewertung von Theorien - Wissenschaft von ihren sozialen Bedingungen gänzlich abstrahiert und entsprechend auch Werteinflüsse aus der Wissenschaft strikt ausschließt. Das betreffende Ideal der Wertfreiheit beruht dabei insbesondere auf zwei neopositivistischen Doktrinen: dem Verifikationismus und der Unterscheidung von Entdeckungs- und Rechtfertigungszusammenhang.

Das verifikationistische Kriterium der Bedeutung postuliert, dass nur bestimmte Sätze überhaupt als kognitiv bedeutungsvoll auszuzeichnen und nur diese Sätze legitime Bestandteile wissenschaftlicher Theorien sind. Diese Sätze seien entweder analytisch wahr (d. h. logisch wahr oder wahr aufgrund der Bedeutungen der in ihnen enthaltenen Terme), oder aber es handele sich um synthetische Sätze, die empirisch überprüfbar (verifizierbar) sind. Die Bedingung der empirischen Prüfbarkeit steht dabei in engem Zusammenhang mit einem erkenntnistheoretischen Fundamentalismus: Bedeutungsvolle synthetische Sätze ließen sich, auch wenn sie theoretische Terme enthielten, auf reine Beobachtungssätze zurückführen, welche durch die Erfahrung bestätigt oder widerlegt werden könnten. Die verifikationistische Bedeutungstheorie impliziert somit die Ablehnung der Möglichkeit synthetischer a priori Erkenntnisse im Sinne der Kantischen Tradition. Sämtliches wissenschaftliches Wissen über die Welt sei dass Ergebnis empirischer Forschung:

Gerade in der Ablehnung der Möglichkeit synthetischer Erkenntnis a priori besteht die Grundthese des modernen Empirismus. Die wissenschaftliche 
Weltauffassung kennt nur Erfahrungssätze über Gegenstände aller Art und die analytischen Sätze der Logik und Mathematik. (Verein Ernst Mach 1929, S. 307)

Der Verifikationismus richtet sich in erster Linie gegen metaphysische Spekulationen, die so als Pseudoprobleme diskreditiert werden. Deren Legitimation in einer wissenschaftlichen Philosophie, als welche sich der Neopositivismus versteht, wird entsprechend verneint. Die Aufgabe einer solchen Philosophie sei nicht das Aufstellen philosophischer Doktrinen, sondern die Klärung der hergebrachten philosophischen Rätsel durch eine logische Analyse der Sprache. Diese Analyse führe dazu, entweder den Scheincharakter dieser Rätsel, sprich ihre kognitive Sinnlosigkeit, aufzudecken oder aber sie in empirische Probleme zu überführen, die dann wiederum Gegenstand der Erfahrungswissenschaften würden (vgl. ebd., S. 305 sowie exemplarisch auch Carnap 1932).

Die Methode der logischen Analyse soll dabei nicht nur die Überwindung der Metaphysik ermöglichen, sondern auch zu dem Ziel einer Vereinheitlichung der Wissenschaft beitragen. Durch die Analyse der Sprache, in der wissenschaftliche Theorien verfasst sind, soll letztlich ein „neutrale[s] Formelsystem, eine[r] von den Schlacken der historischen Sprachen befreite[n] Symbolik“" (Verein Ernst Mach 1929, S. 305) geschaffen werden, in dem alle Wissenschaften repräsentiert und so ineinander übersetzbar gemacht werden können. Hier findet sich das Ideal des aperspektivischen bzw. intersubjektiv gültigen Wissens als Charakteristikum und Ziel der Wissenschaft wieder: Der Neopositivismus sucht nach formalen Antworten, die lediglich auf den Mitteln der Logik beruhen und die daher unabhängig von jedem disziplinären, historischen oder politischen Kontext sowie aus diesen Kontexten eventuell resultierenden Werteinflüssen sind.

Nicht nur die Methode der logischen Analyse, auch die verifikationistische Bedeutungstheorie betrifft dabei die Frage der Wertfreiheit. Wertaussagen sind offensichtlich nicht analytisch wahr. Andererseits sind sie auch nicht auf Beobachtungssätze reduzierbar, da es sich eben nicht um empirische Sätze handelt (bzw. ihre Rückführung auf solche dem oben beschriebenen naturalistischen Fehlschluss gleichkäme). Die logische Trennung präskriptiver und deskriptiver Sätze (3) wird im Neopositivismus auf die Spitze getrieben: Werturteile sind nicht nur nicht aus empirischen 
Sätzen ableitbar, sie sind nicht einmal kognitiv sinnvoll und damit auch kein möglicher Bestandteil wissenschaftlicher Theorien. Zudem steht hier die Trennung des Seins vom Sollen in enger Verbindung zu der Idee der methodischen Kontrolle (4) in der Wissenschaft: das Sollen ist für die Wissenschaft irrelevant, da diese sich über die Möglichkeit der empirischen Überprüfung definiert.

Die Darstellung des Neopositivismus ist hier bewusst einfach gehalten, da sie nur als Hintergrund für die historische Entwicklung des Wertfreiheitsideals dient. Natürlich gab es zahlreiche Kritiken und Probleme, mit denen sich die Neopositivisten auch auseinandersetzten, so dass es eigentlich inadäquat ist, diese als statische und einheitliche Position zu beschreiben. Auch gab es eklatante Unterschiede zwischen einzelnen Vertretern des logischen Empirismus, die nicht zuletzt deren Einstellungen zu Fragen der Relevanz von Werten für die Wissenschaft betreffen. Besonders in den letzten Jahren hat es eine Reihe von Untersuchungen gegeben, die diesbezüglich ein differenzierteres Bild zeichnen. Oftmals wird dabei unterschieden zwischen einem rechten Flügel, der eher klassische Positionen vertritt (z. B. Carnap oder Schlick) und einem linken Flügel (z. B. Neurath, Franck), der, im Rückgang auf Duhem, für eine legitime Rolle sozialer Werte bei der Theoriewahl argumentiert (vgl. z. B. Cartwright et al. 1996; Howard 2006, 2009; Neurath 1913; Reisch 2005; Uebel 2000). Insgesamt wird ein stärkeres Gewicht auf den aufklärerischen Impetus der wissenschaftlichen Weltauffassung und Philosophie gelegt, welche für die Neopositivisten durchaus nicht nur abstrakte, sondern auch politisch relevante Ziele waren. Die wissenschaftliche Weltauffassung wurde explizit in der Tradition der Aufklärung verortet und im Bezug zur gesellschaftlichen Situation gedacht:

So zeigen z. B. die Bestrebungen zur Neugestaltung der wirtschaftlichen und gesellschaftlichen Verhältnisse, zur Vereinigung der Menschheit, zur Erneuerung der Schule und der Erziehung einen inneren Zusammenhang mit der wissenschaftlichen Weltauffassung; es zeigt sich, dass diese Bestrebungen von Mitgliedern des Kreises bejaht, mit Sympathie betrachtet, von einigen auch tatkräftig gefördert werden. (Verein Ernst Mach 1929, S. 304)

Der wissenschaftlichen Weltauffassung wird hier selbst ein Wert zugeschrieben, der mit sozialen Werten in engem Zusammenhang steht und die aufklärerische Position zum Ausdruck bringt, dass rationales und klares 
Denken letztlich zu einer Verbesserung der gesellschaftlichen Situation führt. Dieser aufklärerische Wert, der den Neopositivismus motiviert, ist den meisten seiner Vertreter zufolge jedoch keiner, der die logische Analyse der Sprache selbst beeinflusst, sondern steht außerhalb dieser. Diese Analyse könne wertfrei durchgeführt werden, da der oberste Wert, zu dessen Verwirklichung sie beitragen soll, auf ihre konkrete Ausgestaltung keinen Einfluss nehme.

Hier kommt eine Unterscheidung zwischen einem Innen und Außen der Wissenschaft zum Ausdruck, die ihre Ausbuchstabierung in der neopositivistischen Unterscheidung von Entdeckungs- und Rechtfertigungszusammenhang findet. Wissenschaft findet nicht in einem Vakuum statt, sondern ist als sozialer Prozess Teil der Gesellschaft. Dieses soziale Umfeld sei jedoch irrelevant dafür, was den Kern der Wissenschaft ausmache: die Rechtfertigung. Hiermit wird die Prüfung der Gültigkeit von Theorien bezeichnet, die über ihre Akzeptanz oder Zurückweisung entscheidet. Kriterien der Gültigkeit seien dabei wissenschaftsintern (etwa, ob eine Theorie sich empirisch bestätigen lässt oder nicht) und unabhängig von Vorstellungen darüber, wie die Welt sein sollte. Derartige Vorstellungen hätten im Rechtfertigungszusammenhang nichts zu suchen, spielten aber eine mögliche Rolle bei der Entdeckung, d. h. bei der Selektion von Themen und Hypothesen oder auch bei der Anwendung von Theorien. ${ }^{16}$

Die Kontextunterscheidung setzt das Modell der hypothetischdeduktiven Prüfung voraus, das sich seit dem 19. Jahrhundert als Reaktion auf die Entwicklung der Wissenschaften durchgesetzt hat, welche zunehmend (zeitgenössisch) unbeobachtbare Entitäten in ihre Erklärungen empirischer Phänomene integrierten (z. B. Atome in der Chemie, vergangene Prozesse in der Geologie oder den Äther im Elektromagnetismus.) Diese Entwicklungen ließen sich mit einer Auffassung wissenschaftlicher

16 Die Interpretation der Kontextunterscheidung, insbesondere ihrer Formulierung durch Reichenbach (vgl. Reichenbach 1938, S. 1-16), ist weitaus kontroverser als hier dargestellt; verschiedene Deutungen dieser Unterscheidung sowie die Plausibilität einer Unabhängigkeit der Rechtfertigung von Werten in anderen Zusammenhängen werden jedoch in Kapitel 9 noch ausführlich diskutiert. An dieser Stelle soll nur dargestellt werden, wie die Kontextunterscheidung in Diskussionen um die Wertfreiheit der Wissenschaft häufig verwendet wird und wie ihre Akzeptanz zu einer bestimmten Form des Wertfreiheitsideals führte. 
Gesetze als induktiv gewonnenen empirischen Generalisierungen in der Tradition Bacons nicht adäquat beschreiben, sondern erforderten eine größere Rolle der Hypothesenbildung. Im hypothetisch-deduktiven Modell ist die Hypothesenbildung frei und kann legitimerweise durch politische Werthaltungen, metaphysische Ansichten oder religiöse Überzeugungen beeinflusst sein. Aus diesen Hypothesen werden empirische Konsequenzen abgeleitet, die überprüft werden. Das Ergebnis dieser Prüfung ist unabhängig von den anfänglichen Werteinflüssen bzw. ist nur dann objektiv, wenn diese Unabhängigkeit gewährleistet ist. Bestätigte Hypothesen oder Theorien wiederum können zu Anwendungen mit sozialen Konsequenzen führen, was jedoch Gegenstand politischer, nicht wissenschaftlicher Entscheidungen istDurch die Kontextunterscheidung werden diejenigen Elemente des Wertfreiheitsideals bestimmt, die für die Objektivität der Wissenschaft entscheidend sind: der Ausschluss des Sollens durch die methodengeleitete Überprüfung von Theorien. Die Fragen der Reinheit sowie Neutralität der Wissenschaft sind diesbezüglich irrelevant - auch Forschung, über deren Direktion anhand politischer Zielvorstellungen entschieden wird und deren Ergebnisse zu politischen Zwecken verwendet werden, kann im relevanten Sinn wertfrei sein. Die Gültigkeit dieser Ergebnisse bleibt von Werteinflüssen unberührt, wenn es sich um gute Wissenschaft handelt.

Im Gegensatz zur verifikationistischen Bedeutungstheorie hat die Kontextunterscheidung das Wertfreiheitsideal nachhaltig geprägt und wird auch heute noch von seinen Verteidigern angeführt, um zwischen Legitimität und Illegitimität von Werteinflüssen in der Wissenschaft zu differenzieren (vgl. z. B. Koertge 2000, S. 50 ff.). Die aktuellen Debatten um das Wertfreiheitsideal drehen sich vor allem um die Wertfreiheit der Rechtfertigung und setzen oftmals voraus, dass Werteinflüsse in Entdeckung oder Anwendung von der Frage der Akzeptanz von Theorien getrennt werden können (und deshalb für die Objektivität der Wissenschaft unerheblich sind). Im folgenden Kapitel soll nun präzisiert werden, in welcher Form das Wertfreiheitsideal heute diskutiert wird. 